\title{
GLOBAL NORM-RESIDUE MAP OVER QUASI-FINITE FIELD
}

\author{
D. S. RIM and G. WHAPLES
}

Dedicated to the memory of Professor Tadasi Nakayama

A field $k$ is called quasi-finite if it is perfect and if $G_{k} \approx \hat{Z}$ where $G_{k}$ is the Galois group of the algebraic closure $k_{c}$ over $k$ and $Z$ is the completion of the additive group of the rational integers. The classical reciprocity law on the local field with finite residue field is well-known to hold on local fields with quasi-finite residue field ([4], [5]). Thus it is natural to ask if the global reciprocity law should hold in the ordinary sense (see $\S 1$ below) on the function-fields of one variable over quasi-finite field. We consider here two basic prototypes of non-finite quasi-finite fields:

(a) field $k$ of non-zero characteristic which is algebraic over the prime subfield $k_{0}$ and has a finite $p$-primary degree for all prime $p$, i.e., $\left[k: k_{0}\right]=\prod_{\nu} p^{\nu p}$ with $\nu_{p}<\infty$ for all $p$.

(b) The formal power-series field of one variable over an algebraically closed field of characteristic zero. In this note we show that the reciprocity law holds in the case (a) whereas it is not so for the case (b). Indeed we show that, for a function-field of one variable of positive genus over the field of type (b), there always exists a non-trivial (abelian) extension in which every prime divisor splits completely, i.e., an extension which can not be distinguised locally.

1. Let $k$ be a quasi-finite field. Given a fixed generator $\sigma$ of the Galois group $G_{k}$, we obtain the identification $\widetilde{\sigma}: \chi\left(G_{k}\right) \stackrel{\approx}{\longrightarrow} Q / Z$ given by $\chi \rightarrow \chi(\sigma)$, where $\chi\left(G_{k}\right)$ denotes the character group of $G_{k}$. Therefore if $L$ is a local field with the residue field $k$, then we obtain Hasse invariant $\operatorname{inv}_{L}: B(L) \stackrel{\approx}{\rightarrow} Q / Z$ which is the composite of two isomorphisms $B(L) \stackrel{\underset{\sim}{\longrightarrow}}{\longrightarrow} \chi\left(G_{k}\right) \stackrel{\underset{\sim}{\longrightarrow}}{\longrightarrow} Q / Z$ where $B(L)$ is the Brauer group of $L$. In turn we obtain the norm residue map $\left({ }^{*}, L\right): L^{*} \rightarrow G_{L}^{a}$

Received June 23, 1965. 
which is characterized by $\chi(a, L)=\operatorname{inv}_{L}(a, \chi)$ for all $\chi \in \chi\left(G_{L}\right)$, where $(a, \chi)$ is the element of $B(L)$ represented by the cup-product, and $G_{L}^{a}$ is the Galois group of the maximal abelian extension of $L$ over $L$. (Thus the local norm residue map depends only on the choice of a generator of the Galois group of the residue field). It follows from the local class field theory that $(*, L)$ induces the isomorphism $(*, M / L): L^{*} / N M^{*} \stackrel{\approx}{\longrightarrow} G(M / L)$ for all finite abelian extensions $M>L$, where $N=N_{M / L}$ stands for the norm mapping. Now let $K$ be a function-field of one variable over $k$. For each prime divisor $p$ of $K$ (which is trivial on $k$ ) the local completion $K_{p}$ is a local field whose residue field $\bar{K}_{p}$ is finite algebraic over $k$. Therefore $\bar{K}_{p}$ is also quasi-finite and we shall agree to choose $\sigma^{d_{p}}\left(d_{p}=\left[\bar{K}_{p}: k\right]\right)$ as a generator of $G_{\bar{K}_{p}}$. Thus we have local norm residue map $\left(*, K_{p}\right): K_{p}^{*} \rightarrow G_{K_{p}}^{a}$ for all $p$, and in turn we obtain the global norm residue map $(*, K): J_{K} \rightarrow G_{K}^{a}$ by $(\alpha, K)=\prod_{p}\left(\alpha_{p}, K_{p}\right)$ which is characterized by $\chi(\alpha, K)=\operatorname{inv}_{K}(\alpha, \chi)=\sum \operatorname{inv}_{K_{\gamma}}\left(\alpha_{p}, \chi\right)$ for all $\chi \in \chi\left(G_{K}\right)$, where $J_{K}$ is the group of idèles of $K$. However, it follows easily from Tzen's theorem and the product formula that the norm residue map is trivial on the principal idèles and hence we have $\left({ }^{*}, K\right): C_{K} \rightarrow G_{K}^{a}$ where $C_{K}$ is the idèle class group of $K$. We shall say that the reciprocity law holds over $k$ if, for any function-field of one variable $K$ over $k$, the norm residue map induces the isomorphism (*, $L / K): C_{K} / N C_{L} \rightarrow G(L / K)$ for all finite abelian extensions $L>K$. Thus the reader should observe that we are concerned with the reciprocity with respect to the norm-residue map on the idele class group and not an abstract class formation.

THEOREM 1. Let $K$ be a function-field of one variable over a quasi-finite field $k$, and let $\widetilde{K}=k_{c} K$ where $k_{c}$ is the algebraic closure of $k$. Then we have the exact sequence

$$
0 \rightarrow H^{1}\left(G_{k}, E(\widetilde{K})\right) \rightarrow B(K) \rightarrow \sum_{p} B\left(K_{p}\right) \stackrel{\text { inv }}{\longrightarrow} Q / Z \rightarrow 0
$$

where $E(\widetilde{K})$ is the divisor class group of $\widetilde{K}$. If $H^{1}\left(G_{k}, E(\widetilde{K})\right)=0$ for all function-fields $K$ of one variable over $k$, then the reciprocity law holds over $k$.

Proof. Consider the exact sequences

$$
\begin{aligned}
& 0 \rightarrow P(\tilde{K}) \rightarrow D(\tilde{K}) \rightarrow E(\tilde{K}) \rightarrow 0 \\
& 0 \rightarrow k_{c}^{*} \rightarrow \widetilde{K}^{*} \rightarrow P(\widetilde{K}) \rightarrow 0
\end{aligned}
$$


where $D(\widetilde{K}), P(\widetilde{K})$ are the group of divisors, principal divisors resp. Since $G_{k} \cong \hat{Z}$, we have scd $G_{k}=2([3])$ and hence we obtain the exact sequences

$\left(^{*}\right)^{\cdot} \quad H^{1}\left(G_{k}, D(\tilde{K})\right) \rightarrow H^{1}\left(G_{k}, E(\tilde{K})\right) \rightarrow H^{2}\left(G_{k}, P(\widetilde{K})\right) \rightarrow$

$H^{2}\left(G_{k}, D(\widetilde{K})\right) \rightarrow H^{2}\left(G_{k}, E(\widetilde{K})\right) \rightarrow 0$

and

$H^{2}\left(G_{k}, k_{c}^{*}\right) \rightarrow H^{2}\left(G_{k}, \tilde{K}^{*}\right) \rightarrow H^{2}\left(G_{k}, P(\widetilde{K})\right) \rightarrow 0$.

Since $k$ is a quasi-finite field, we have $H^{2}\left(G_{k}, k_{c}^{*}\right)=0$ and hence $B(K) \simeq H^{2}\left(G_{k}\right.$, $\left.\widetilde{K}^{*}\right) \simeq H^{2}\left(G_{k}, P(\tilde{K})\right)$. Furthermore $D(\widetilde{K})=\sum_{p} \operatorname{Hom}_{Z_{\left(D_{p}\right)}}\left(Z\left(G_{k}\right), Z\right)$, where $D_{p}$ is the decomposition subgroup of the prime divisor $p$, entails $H^{1}\left(G_{k}, D(\widetilde{K})\right)=$ $\sum_{p} H^{1}\left(D_{p}, Z\right)=0$. Therefore the exact sequence $\left(^{*}\right)$ becomes

$$
\left({ }^{* *}\right) \quad 0 \rightarrow H^{1}\left(G_{k}, E(\widetilde{K})\right) \rightarrow B(K) \rightarrow H^{2}\left(G_{k}, D(\widetilde{K})\right) \rightarrow H^{2}\left(G_{k}, E(\widetilde{K})\right) \rightarrow 0 .
$$

Now let us consider the exact sequence $0 \rightarrow E_{0}(\widetilde{K}) \rightarrow E(\widetilde{K}) \stackrel{d}{\longrightarrow} Z \rightarrow 0$ where $d$ is the degree map. Since $E_{0}(\tilde{K})$ is a divisible group, we get $H^{2}\left(G_{k}, E_{0}(\tilde{K})\right)=0$ ([3]) and hence we obtain the isomorphism $\tilde{d}: H^{2}\left(G_{k}, E(\tilde{K})\right) \rightarrow H^{2}\left(G_{k}, Z\right)$. Now the composite map $H^{2}\left(D_{p}, Z\right) \rightarrow \sum_{p} H^{2}\left(D_{p}, Z\right)=H^{2}\left(G_{k}, D(\widetilde{K})\right) \rightarrow H^{2}\left(G_{k}\right.$, $E(\widetilde{K})) \stackrel{\tilde{d}}{\longrightarrow} H^{2}\left(G_{k}, Z\right)$ is nothing but the corestriction map ([3]), and hence the composite map $H^{2}\left(G_{k}, D(\widetilde{K})\right) \rightarrow H^{2}\left(G_{k}, E(\widetilde{K})\right) \stackrel{\widetilde{d}}{\rightarrow} H^{2}\left(G_{k}, Z\right) \stackrel{\widetilde{\sigma}}{\rightarrow} Q / Z$ is given by $\sum_{p} \chi_{p} \rightarrow \sum_{p} \chi_{p}\left(\sigma^{d_{\nu}}\right)$ where $d_{p}=\left[G_{k} ; D_{p}\right]$. Consequently the diagram

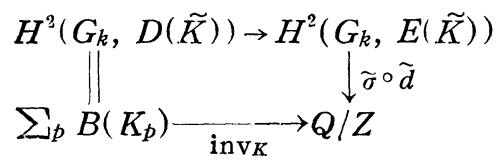

is commutative. It follows that

$$
\left.0 \rightarrow H^{1}\left(G_{k}, E(\tilde{K})\right) \rightarrow B(K) \rightarrow \sum{ }_{p} B, K_{p}\right) \stackrel{\mathrm{inv}_{K}}{\rightarrow} Q / Z \rightarrow 0
$$

is exact. Now assume that $H^{1}\left(G_{k}, E(\widetilde{K})\right)=0$ for every function-field $K$ of one variable over $k$, and let $L>K$ be any Galois extension. Then the exact sequence $0 \rightarrow B(L) \rightarrow \sum_{p} B\left(L_{p}\right) \stackrel{\text { inv } L}{\longrightarrow} Q / Z \rightarrow 0$ of $G=G(L / K)$-modules gives us the exact sequence $0 \rightarrow B(L)^{G} \rightarrow\left(\sum_{p} B\left(L_{p}\right)\right)^{G} \rightarrow Q / Z \rightarrow H^{1}(G, B(L)) \cdots$. Now the exact commutative diagram

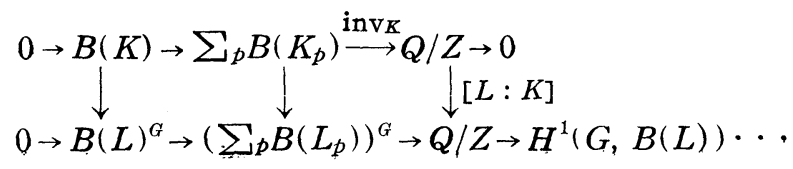


gives us ([1] p. 40) the exact sequence

$$
\begin{aligned}
& 0 \rightarrow H^{2}\left(G(L / K), L^{*}\right) \rightarrow H^{2}\left(G(L / K), J_{L}\right) \stackrel{\stackrel{\text { inv } K}{\longrightarrow} Z / n Z \rightarrow}{B}, \\
& B(L)^{G} / \operatorname{Im}(B(K) \rightarrow B(L)) \cdots \text { where } n=[L: K] .
\end{aligned}
$$

On the other hand, the exact sequence $0 \rightarrow L^{*} \rightarrow J_{L} \rightarrow C_{L} \rightarrow 0$ gives us the exact sequence

$$
\begin{gathered}
0 \rightarrow H^{1}\left(G(L / K), C_{L}\right) \rightarrow H^{2}\left(G(L / K), L^{*}\right) \rightarrow H^{2}\left(G(L / K), J_{K}\right) \rightarrow \\
H^{2}\left(G(L / K), C_{L}\right) \rightarrow H^{3}\left(G(L / K), L^{*}\right) . \text { Now suppose that }
\end{gathered}
$$

$L>K$ is a cyclic extension. Then the exact sequence $0 \rightarrow H^{2}\left(G(L / K), L^{*}\right) \rightarrow$ $B(K) \rightarrow B(L) \stackrel{G(L / K)}{\longrightarrow} H^{3}\left(G(L / K), L^{* /}\right) \rightarrow \cdots$ entails that $B(K) \rightarrow B(L)^{G}$ is an epimorphism, and hence (1) becomes

$$
0 \rightarrow H^{2}\left(G(L / K), L^{*}\right) \longrightarrow H^{2}\left(G(L / K), J_{L} \stackrel{\text { inv } K}{\longrightarrow} Z / n Z \rightarrow 0\right.
$$

Comparing (1)' and (2), we conclude that for all cyclic extensions $L>K$ we have $H^{1}\left(G(L / K), C_{L}\right)=0$ and $\left|H^{2}\left(G(L / K), C_{L}\right)\right|=[L: K]$. In particular it follows that for any Galois extension $L>K$ there exists at least one prime divisor in $K$ which does not split completely in $L$. Consequently $(*, L)$ : $C_{K} \rightarrow G_{K}^{a}$ induces the epimorphism $\left({ }^{*}, L / K\right): C_{K} / N C_{L} \rightarrow G(L / K)$ for all cyclic extensions $L>K$ of prime degree, and hence $(*, L / K)$ is the epimorphism for all finite abelian extensions $L>K$. On the other hand, $\left|H^{2}\left(G(L / K), C_{L}\right)\right|=$ $[L: K]$ for all cyclic extensions $L>K$ entails that $\left|H^{0}\left(G(L / K), C_{L}\right)\right| \leq[L: K]$ for all solvable extensions, and hence $(*, L / K): C_{K} / N C_{L} \rightarrow G(L / K)$ is an isomorphism for all finite abelian extension $L>K$. This completes our proof.

COROLlary. The reciprocity law holds over a quasifinite field of type a).

Proof. Let $k$ be a quasi-finite field of type a), and let $K$ be a functionfield of one variable over $k$. Then we can find a finite subfield $k_{0}$ and a function-field $K_{0}$ over $k_{0}$ such that $K=k K_{0}$. It follows from Hasse's norm theorem that $H^{1}\left(G_{k}, E(\widetilde{K})\right)=0$ for all finite extensions $k_{1}>k_{0}$. Since $K=k K_{0}=\bigcup_{j} k_{j} K_{0}$ where $k>k_{j}>k_{0}$ runs through intermediate finite fields, we obtain that $H^{1}\left(G_{k}\right.$, $E(\tilde{K}))=\lim _{\rightarrow} H^{1}\left(G_{k j}, E(\widetilde{K})\right)=0$.

2. The reciprocity law fails over the quasi-finite field of type $b$ ). Indeed, there exists an abelian extension $L \supsetneqq K$ of function fields of one variable over 
the quasi-finite field of type (b), in which every prime of $K$ splits completely in $L$, i.e., $L, K$ cannot be distinguished locally. One way to see this is to construct an elliptic curve whose index is bigger than one; if ind $K>1$ with the constant field $k$, then $K k^{\prime}>K$ provides such an example, if we set $\left[k^{\prime}: k\right]$ $=$ ind $K$. It is not difficult to construct such a field following the homological interpretation of principal homogeneous spaces (see [2]). Our theorem below, which is of interest in itself, provides a different kind of example, namely an extension $L \supsetneqq K$ which is linearly disjoint from the constant field extension and in which every prime in $K$ splits completely in $L$.

Let $k$ be a quasi-finite field of type (b), or more generally let $k=k_{0}((t))$ where $k_{0}$ is an algebraically closed field of any characteristic. If $K_{0}$ is a function-field of one variable over $k_{0}$, then $K=k K_{0}$ is a function-field of one variable over $k$. Let us denote by $\Sigma_{K}, \Sigma_{K_{0}}$ the set of prime divisors of $K, K_{0}$ over $k, k_{0}$ resp. We define a map $\pi: \Sigma_{K} \rightarrow \Sigma_{K_{0}}$ as follows: let $v$ be a non-trivial discrete valuation on $K$ over $k$. If its restriction on $K_{0}$ is non-trivial, we set $\pi(v)=$ the restriction of $v$ on $K_{0}$. If the restriction of $v$ on $K_{0}$ is trivial, then $v$ induces the inclusion map $i_{v}: K_{0} \rightarrow O_{v}$ where $O_{v}$ denotes the residue field of the valuation ring of $v$. Since $O_{v}$ is a finite extension of $k=k_{0}((t))$ which is complete under $t$-valuation, $O_{v}$ is provided with the $t$-valuation which is the unique extension of the $t$-valuation on $k$, and its restriction on $K_{0}$ through the embedding $i_{v}$ is non-trivial. (Indeed, let $O_{v}=k_{0}((T))$ where $T$ is a prime element with respect to $t$-valuation, and set, for each $x \in K_{0}, i_{v}(x)=\Sigma a_{i}(x) T^{i}$ with $a_{i}(x) \in k_{0}$. If $i_{v}(x)$ is a $t$-unit then $i_{v}\left(x-a_{0}(x)\right)$ is not a $t$-unit since $i_{v}$ is $k_{0}$-linear map.) We then define $\pi(v)$ to be the restriction of the $t$-valuation through the embedding $i_{v}: K_{0} \rightarrow O_{v}$. We observe here that the embedding $i_{v}$ : $K_{0} \rightarrow O_{v}$ induces the unique extension $i_{v}: \hat{K}_{0} \rightarrow O_{v}$ where $\hat{K}_{0}$ denotes the completion of $K_{0}$ under the valuation $\pi(v)$. We shall denote the degree $\left[O_{v}: \hat{K}_{0}\right]$ by $\delta(v)$. We also observe that there is a canonical map $\varepsilon: \Sigma_{K_{0}} \rightarrow \Sigma_{K}$ such that $\pi \cdot \varepsilon=$ identity, where $\varepsilon(v)$ is the unique extension of $v$ to $K$ which is trivial on $k$.

Now let $L_{0}>K_{0}$ be a finite extension. This gives rise to a finite extension $L>K$ under the constant field extension from $k_{0}$ to $k$, and we obtain the commutative diagram 


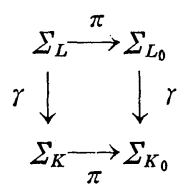

Lemma. Let $v$ be in $\Sigma_{K}$.

(1) If $v=\varepsilon \pi(v)$, then $v$ splits completely in $L>K$ if and only if $\pi(v)$ splits completely in $L_{0}>K_{0}$.

(2) If $v \neq \varepsilon \pi(v)$, then $v$ splits completely in $L>K$ if and only if $\delta(v)$ is divisible by the ramification indices of $\pi(v)$ in $L_{0}>K_{0}$.

Proof. (1) $\sharp \gamma^{-1}(v)=\# \gamma^{-1} \varepsilon \pi(v)=\# \gamma^{-1} \pi(v)$

(2) Let $O_{v}$ be the valuation ring of $v . \quad L_{0} \otimes O_{0} O_{v}$ is an integral domain since $L_{0}, K$ are linearly disjoint over $K_{0}$, and furthermore the Jacobson-radical of $L_{0} \otimes O_{v}$ is a principal ideal. It follows that $L_{0} \underset{K_{0}}{\otimes} O_{v}$ is the integral closure of $O_{v}$ in $L$. Now $L_{0} \underset{K_{0}}{\otimes} O_{v}=L_{0} \underset{K_{0}}{\otimes} O_{v} \underset{\hat{K}_{0}}{\otimes} O_{v}=\left(\hat{L}_{0}^{(1)} \oplus \hat{L}_{0}^{(2)} \oplus \cdots \oplus \hat{L}_{0}^{(q)}\right) \underset{\hat{K}_{0}}{\otimes} O_{v}$ where $\hat{K}_{0}$ is the completion of $K_{0}$ under the valuation $\pi(v)$ and $\hat{L}_{0}^{(1)}, \ldots, \hat{L}_{0}^{(q)}$ are the completions of $L$ under the extensions of $\pi(v)$. Consequently $v$ splits completely in $L$, i.e., $L_{0} \otimes O_{0} O_{v}$ is $[L: K]$-copies of $O_{v}$ if and only if there exist $K_{0}$-imbeddings $L_{0}<O_{v}$ for all $j$, i.e., if and only if $\delta(v)=\left[O_{v}: \hat{K}_{0}\right]$ is divisible by $\left[\hat{L}_{0}^{(j)}: \hat{K}_{0}\right]$ for all $j$. However, $\left[\hat{L}_{0}^{(j)}: \hat{K}_{0}\right]$ is nothing but the ramification index of the corresponding valuation.

THEOREM 2. Let $v_{0}$ be in $\Sigma_{K_{0}}$.

(1) If $v_{0}$ is unramified in $L_{0} / K_{0}$, then $\pi^{-1}\left(v_{0}\right)$ splits completely in $L / K$.

(2) If $v_{0}$ is ramified in $L_{0} / K_{0}$ then $\pi^{-1}\left(v_{0}\right)$ contains infinitely many primes which do not split completely in $L / K$ as well as infinitely many primes which do split completely in $L / K$.

Proof. (1) This follows immediately from the above lemma. (2) Let us simply denote by $\hat{K}_{0}=k_{0}((T))$ the completion of $K_{0}$ under the valuation $v_{0}$, where $T$ is a fixed prime element taken inside $K_{0}$. For each element $\lambda$ in $k$ with $v_{t}(\lambda)>0$ (where $v_{t}$ is the $t$-valuation on $k$ ), we obtain the $k$-algebra map $\hat{K}_{0} \otimes_{k_{0}} k=k_{0}((T)) \underset{k^{0}}{\otimes} k \rightarrow k$ determined by $T \rightarrow \lambda$ and the continuity. Restricting this map on $K_{0} \otimes k$ we obtain the $k$-algebra map $\varphi_{\lambda}: K_{0} \otimes k \rightarrow k$ such that $\varphi_{\lambda}(T)$ $=\lambda$, and $\varphi_{\lambda}$ determines the valuation $v_{\lambda}$ on $K$ such that $\pi\left(v_{\lambda}\right)=v_{0}$. We observe that $\delta\left(v_{\lambda}\right)=\left[k: \hat{K}_{0}\right]=v_{t}(\lambda)$, and that $v_{\lambda}, v_{\mu}$ are inequivalent valuations of $K$ if 
$\lambda \neq \mu \quad$ Therefore, for any given natural integer $m$, there exist infinitely many primes $v$ in $\Sigma_{K}$ such that $\pi(v)=v_{0}$ and $\delta(v)=m$, and consequently our statement follows from the above lemma.

Corollary. Every prime splits completely in $L>K$ if and only if it is so in $L_{0}>K_{0}$.

Now let $K_{0}$ be a function field of one variable over $k_{0}$ with positive genus. Then $K_{0}$ admits an unramified abelian extension $L_{0} \supsetneqq K_{0}$, and every prime splits completely since the constant field $k_{0}$ is algebraically closed. It follows from the above corollary that every prime splits completely in the abelian extension $L \Varangle K$, and thus the reciprocity law fails over the quasi-finite field of type (b).

\section{BIBLIOGRAPHY}

[1] H. Cartan and S. Eilenberg, Homological Algebra.

[2] S. Lang and J. Tate, Principal homogeneous spaces over abelian varieties, American Journal of Math., vol. 80 (1958), pp. 659-684.

[3] J. P. Serre, Cohomologie Galoisienne, College de France, 1963.

[4] J. P. Serre, Corps Locaux, Hermann, 1962.

[5] G. Whaples, Generalized local class field theory, I Duke Math. Journal, vol. 19 (1952), pp. 505-517.

University of Pennsylvania

Indiana University

University of Notre Dame 